\title{
The Journal of Applied Oral Science and the open science era
}

Dear Readers, Contributors and Reviewers,

In my Editorial debut as the Co-Editor-in-Chief of the Journal of Applied Oral Science (JAOS), I would like to share with you some thoughts and views about the challenges and perspectives of the open science era.

The increase in the generation of scientific data in oral science field is evident and noteworthy, and consequently the dissemination of such new observational and experimental data is fundamental to support or reject new theories, and to support subsequent advances. In this context, the choice of the ideal journal to have the data published currently involves not only the focus in a given knowledge area, ranging from specific niches to broader audiences, but also the concerns in providing a universal access to the data published. Indeed, the open access concept, besides being a desire or a recommendation, became a mandatory condition in the view of some universities, research institutes and funding agencies, which recognize that allowing openness to consult and inquiry is a fundamental step of science advance.

An important point to be considered is the cost of open access. It is obvious that the management of a journal within high standards demands a substantial financial support, which is usually translated into costs for the publishing scientists (costs for publication and additional rates for open access in several instances) and for the audience (journal signatures or individual taxes for constrained access). The huge pressure for publications also expanded the editorial field, whose commercial aspects are a serious concern of some scientific groups.
While the high cost of this process became more evident due to the recent boundaries to research funding engendered by the global financial instability, journals such as the JAOS, counting with the financial support of the University of São Paulo and governmental Brazilian agencies (such as FAPESP, CNPq and CAPES), reinforce its open access and non-profit principles, providing a solid platform for science publication with minimal (or without) costs to the authors.

Moreover, in order to provide the best conditions to disseminate original data in the oral science field, the JAOS still resists to impose major restrictions to manuscripts length, which does not mean unnecessary lengthy papers, but the adequate space to describe and discuss substantial volume of data generated with modern research tools. Also, the recent migration to the ScholarOne system already presented a very positive impact in the journal routine, since the system workflow is widely known for authors and reviewers, and the increasing agility of the review process is acknowledged and appreciated by all of them. Finally, the adoption of the XML tagging will adequate the articles format to the PubMed Central guidelines, and widen the indexing tags, resulting in a higher visibility in different databases.

Therefore, the JAOS editorial team reinforces its commitment with a real open science environment, and one of my major goals as the Co-Editor-in-Chief is to keep and develop this concept in every edition of the journal.

\section{Gustavo Pompermaier Garlet Co-Editor-in-Chief Journal of Applied Oral Science}

